\title{
Targeting Advertisements and Inferring Demographics in the Hospitality Industry
}

\author{
Adam Abrams ${ }^{1}$, Melanie Beckerleg ${ }^{2}$, and Maryia Shpak ${ }^{3}$ \\ ${ }^{1}$ Institute of Mathematics, Polish Academy of Sciences, Warsaw, Poland \\ ${ }^{2}$ University of Oxford, UK \\ ${ }^{3}$ Institute of Mathematics, Maria Curie-Sktodowska University, Lublin, Poland
}

(Communicated to MIIR on 31 October 2021)

Study Group: ECGI 144, 17-22 March, 2019, Polish Academy of Sciences, Warsaw, Poland

Communicated by: Kamil Kulesza

Industrial Partner: RoomSage

Presenter: Piotr Zioło

Team Members: Adam Abrams, Polish Academy of Sciences; Melanie Beckerleg, University of Oxford; Artur Chabowski, Georgia Institute of Technology; Kamil Kulesza ${ }^{4}$, Polish Academy of Sciences; Maryia Shpak, Maria Curie-Skłodowska University, Lublin; Mitsuru Wilson, Polish Academy of Sciences

Industrial Sector: Hospitality

Key Words: Computational advertising, Machine learning, Bayesian networks, Optimization

MSC2020 Codes: 68, 90, 62

\section{Summary}

There are many channels which can be used for advertising - Google Ads, Facebook, Google Hotel Ads, Trivago, some of which allow direct communication with a single person, while other only allow advertising to groups of people. A typical booking process through Google Ads looks as follows: the hotel creates an ad (potentially a personalized ad), a potential client searches for a hotel in Google, the ad is shown to the user, the user clicks on the ad and is redirected to the hotel's website, and there makes a reservation. As the advertiser pays per click, the proper measure of the ad/offer performance is the average value per click. The main problem to be solved can be stated as follows: design hotel clients segmentation techniques coupled with automated offer generation methods allowing to maximize the value per click from an ad campaign.

${ }^{4}$ Corresponding contributor: kamil.kulesza@maths.com.pl 


\section{Contents}

1 Introduction $\quad 4$

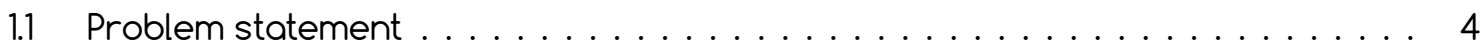

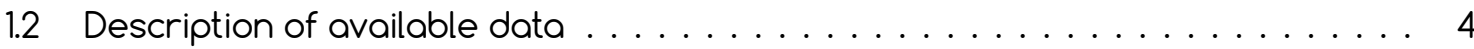

2 Data analysis $\quad 6$

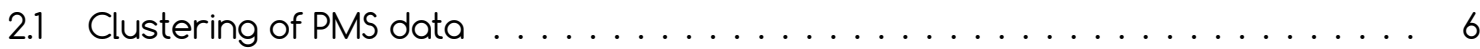

2.2 Time scales in Google data . . . . . . . . . . . . . . . . . . . . . . . . 8

2.3 Segmentation in Google data . . . . . . . . . . . . . . . . . . . . . . . . 10

3 Theoretical model for profit 11

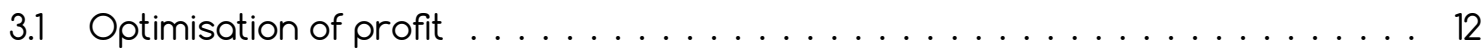

3.2 Hypothetical conversion models . . . . . . . . . . . . . . . . . . . . . 12

3.3 Dynamic pricing . . . . . . . . . . . . . . . . . . . . . . . . . . . . . . 15

4 Decision-making processes 16

4.1 Bayesian updating $\ldots \ldots \ldots \ldots \ldots \ldots \ldots \ldots \ldots \ldots$

4.2 Feedback loop ............................ . 16

$\begin{array}{llr}5 & \text { Further ideas } & 17\end{array}$

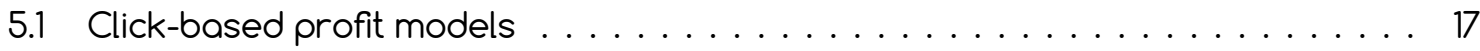

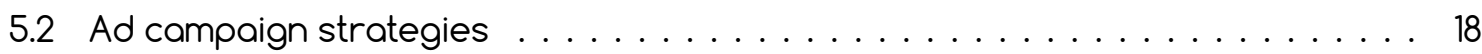

5.3 Automation algorithm . . . . . . . . . . . . . . . . . . . . . . 20

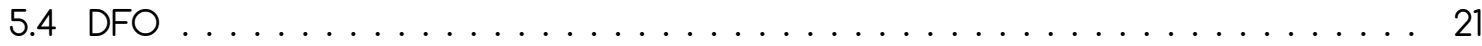

5.5 Hidden Markov Models . . . . . . . . . . . . . . . . . . . . . . . . . . . . 21

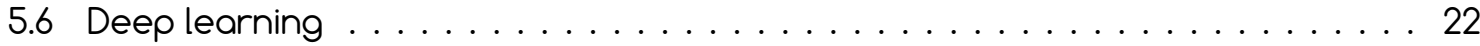

5.7 Recommendation systems approach . . . . . . . . . . . . . . . . . . 23 


\section{Introduction}

\subsection{Problem statement}

The online hotel booking market is now dominated by Online Travel Agents (OTAs), large companies that can facilitate booking rooms at multiple hotels for a commission. In 2017, these agencies accounted for 39\% of the US market share [14], so hotels (especially smaller ones) are forced to use these OTAs unless they can target ads more efficiently to achieve bookings directly. While a small commission is reasonable for the service of drawing customers, many online travel agencies now charge 15-30\% in commissions [6], which may be hidden from the consumer and yet very costly to the hotelier.

Hotels not willing to pay the commission would find value in algorithms to find optimal strategies for advertising campaigns. This is the goal of RoomSage: to provide hoteliers with tools to "drive outstanding growth in direct bookings through advertising while reducing their dependence on online travel agency channels" [23]. The design of advertisements would account for the impact of discounts on conversion rate in online advertising as well as the impact of the demographic composition of clusters of customers.

For the purposes of clarity, in this report we say that segments of the population are based on demographic characteristics (e.g., age, gender, country of origin) while clusters are based on reservation data from a hotel.

\subsection{Description of available data}

\subsubsection{Property Management System (PMS)}

Hotels collect data on individual reservations. This includes details on the booking itself (e.9., dates, rooms, number of guest) and, when possible, demographic information on the guests, such as age, gender, and location of origin.

Reservations are made through a "Property Management System" (PMS), the first of which were Fidelio and Hogatex in the 1980s [16]. What data features are collected presumably differs between various PMS and various hotels. During the ESGI144, data were available from one single hotel in Canada, with most reservations made in 2017 and 2018. 


\subsubsection{Google Adwords daily totals}

Each entry in the maindb.gadw_campaigns_daily table contains the following fields, among others:

- rs_campaign_id and campaign_id - which ad compaign the entry is for (in the sample data, every value in each of these fields is the same for every row);

- date - the date;

- impressions - the number of times users see an ad from this compaign on this day;

- clicks - the number of times users click on ad an from this campaign on this day;

- conversions - an estimate of the number of times users actually book hotel rooms after clicking on an ad from this compaign on this day;

- ctr - the "click through rate" for this compaign, which is the number of clicks divided by the number of impressions;

- conversion_rate - number of conversions divided by number of clicks;

- average_cpc - cost per click;

- cost_per_conversion.

Google does not know with certainty whether a user actually makes a reservation after viewing/clicking on an ad because it does not have access to the actual PMS data; thus the number of conversions is an estimate.

Also, the click through rate and conversion rate in the table are calculated for each individual day. Calculating a conversion rate for a week or other time span requires counting the total click and conversions for that time span and dividing those totals. Since different time scales are used for different purposes (see Section 2.2), the pre-computed rate fields are not especially useful.

\subsubsection{Google Adwords demographic breakdowns}

The maindb.gadw_gender_daily table has a Gender field whose possible values are
- Male
- Female
- Undetermined. 
The maindb.gadw_age_daily table has an Age field whose possible values are

- $18-24$

- 55-64
- 25-34

- 65 or more
- $35-44$

- Undetermined.

The maindb.gadw_gender_age_daily table has both Gender and Age fields.

The maindb.gadw_devices_daily table has a Device field that takes values of
- Desktop
- Mobile
- Tablet
- Other.

Lastly, the maindb.gadw_geography_daily table has fields for Country and Region (e.9., States in the US, Provinces in Canada).

From each of the tables above, one can find the number of impressions, clicks, and conversions for a given category on a given day. For example, in our sample data, the table maindb.gadw_gender_age_daily records that on 21 November 2018 there were 7 impressions, 3 clicks, and 2 conversions made by women aged 55-64.

While the bedb.booking_engine_availability_checks table also has fields labelled age and gender, in the sample data of 4138 rows in this table every single age and gender entry was blank.

\section{Data analysis}

\subsection{Clustering of PMS data}

Machine Learning tasks are broadly categorised as "supervised" or "unsupervised" learning [1]. Supervised learning includes, for example, image recognition. Two common examples of unsupervised learning are separating overlapping audio signals and partitioning data points into related clusters. This latter process is applicable to our project. Figure 1 shows an illustration of what clustering can look like for data with only two features, plotted as $x$ and $y$-coordinates. 

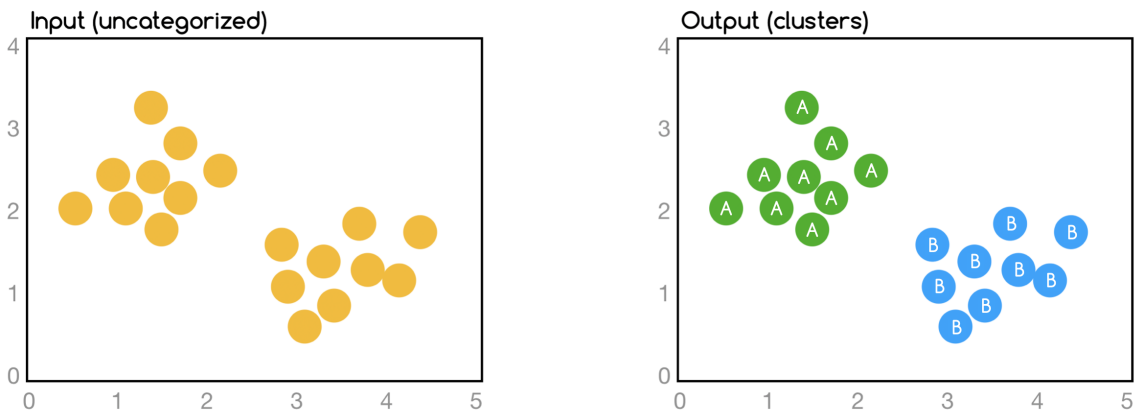

Figure 1: Example of clustering in two-feature data.

Given hotel reservation data, there are established algorithms that can determine "clusters", groups of reservations that ideally share certain characteristics and are worth considering as meaningful units. The clusters analysed in Figure 2 were determined using a $k$-means algorithm (see, for example, [4]).
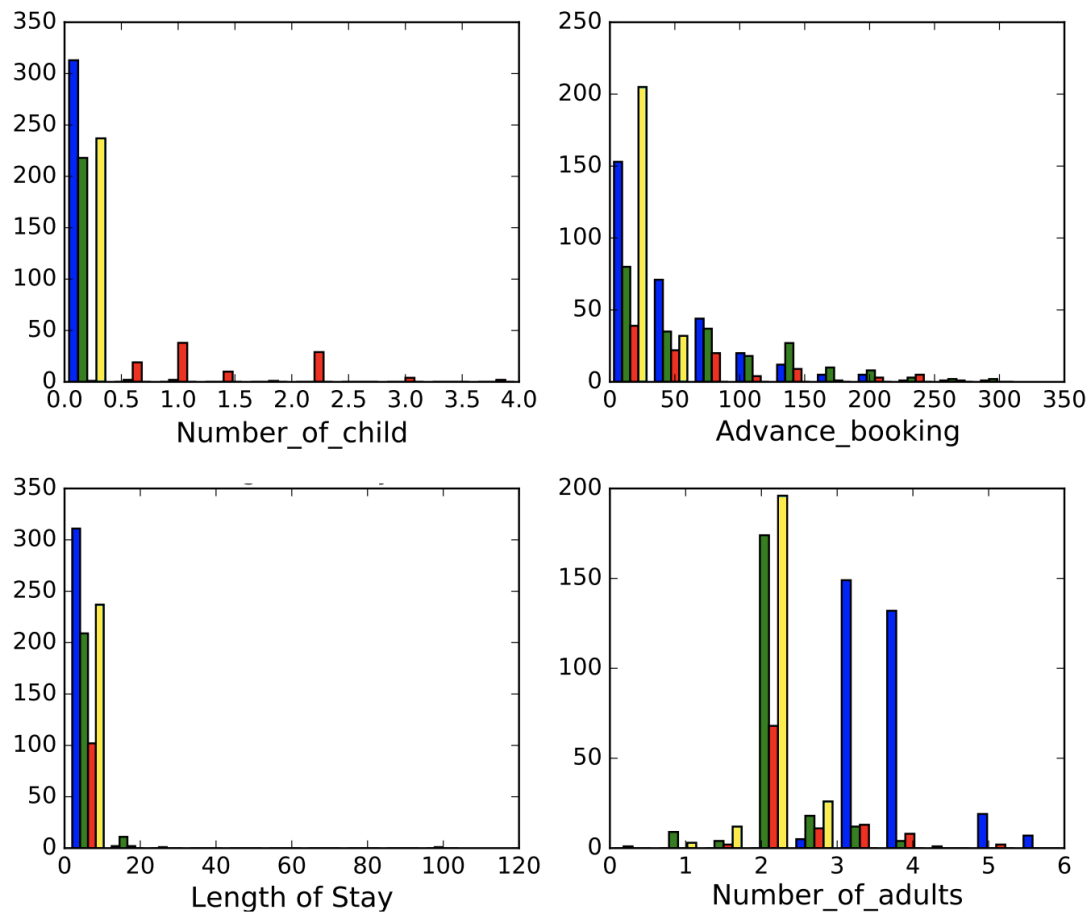

Figure 2: Histograms of various features by cluster (each cluster is a different colour).

For some clusters, we can give a human-language interpretation. For example, the yellow cluster in Figure 2 mostly consists of couples with no children who stayed at the hotel for about two weeks and booked a room close to the date of arriving. After demographic analysis, this cluster could be potentially divided into two clusters of young couples who 
don't have children yet and older couples with grown-up children who don't travel with them anymore.

\subsection{Time scales in Google data}

The data provided by Google Adwords is broken down by day. There is considerable variation from one day to the next, so grouping data by week or by month is significantly more helpful for analysing certain features.

In Figure 3, for example, there is no clearly recognisable pattern when impressions, clicks, and conversions are graphed for each day. However, if we group such data by week (see Figure 4), patterns emerge. In particular, it seems that the numbers of impressions and clicks are proportional within each week.

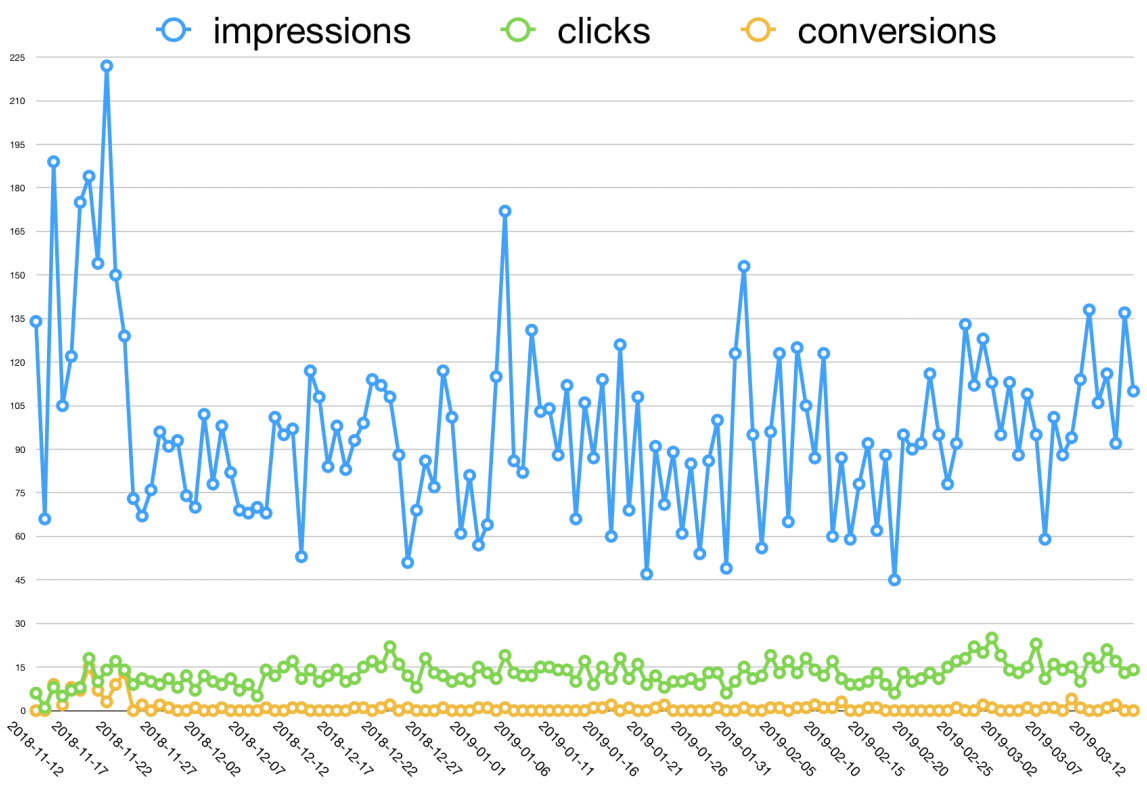

Figure 3: Impressions, clicks and conversions by day.

To verify this apparent relation between impressions and clicks (and to calculate the proportionality constant, which in this case is called the "click through rate"), we divide the number of clicks in a given week by the number of impressions in the same week and compute the average of all such ratios (see left of Figure 5), getting approximately 0.1436. Finally, plotting the number of impressions scaled by 0.1436 and the number of clicks shows how these two data sets line up quite well (right of Figure 5). This confirms that the ratio between impressions and click does not really depend on time, and, at least over the time scale of this particular campaign, suggests that the moment when to show the ads in Google in order to maximise the number of people who click on them is irrelevant. 


O. impressions clicks $\quad 0$ conversions

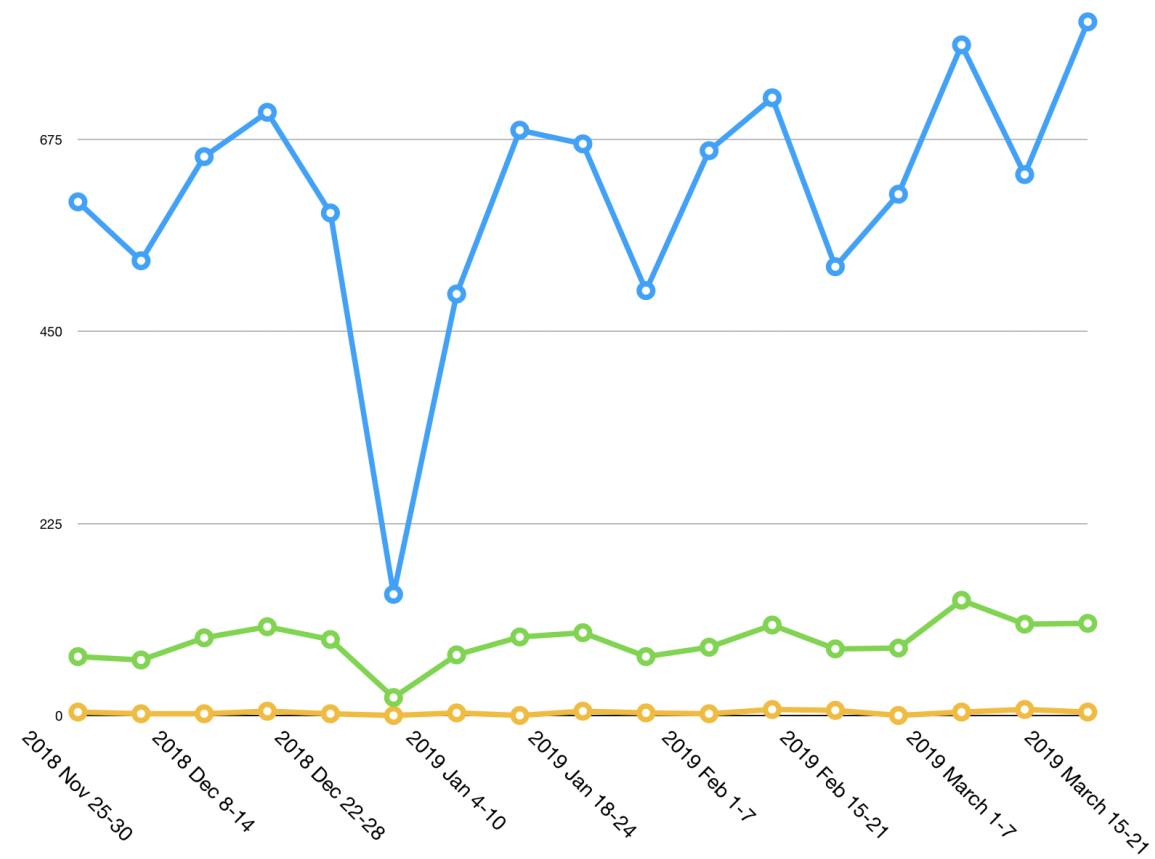

Figure 4: Impressions, clicks and conversions by weeks.
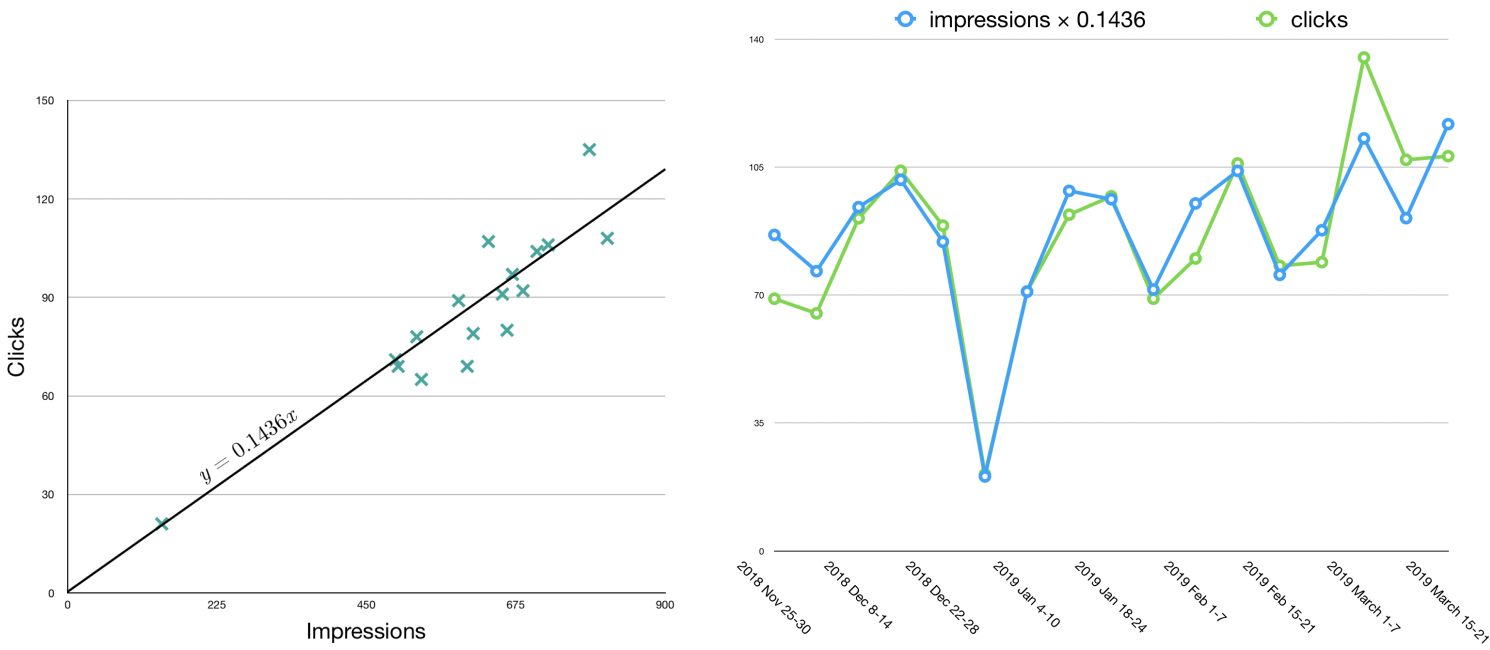

Figure 5: Left: Determining click-through rate with linear regression. Right: Scaled impressions graphed along with actual clicks. 


\subsection{Segmentation in Google data}

As described in Section 1.2.3, the Google Adwords data includes demographic data, including age and gender. It is worth investigating how these features do or don't relate to numbers of impressions, numbers of clicks, and click through rates.

Figure 6 shows that, while ads are seen by men and women with more or less the same frequency (impressions, on the left side of the figure), the men are slightly more likely to click on the adds. However, there are a significant number of users whose genders are unknown in this data set, so that distinction between men and women only holds if we assume that genders of those uncategorised data points distribute similarly to the gendered data.
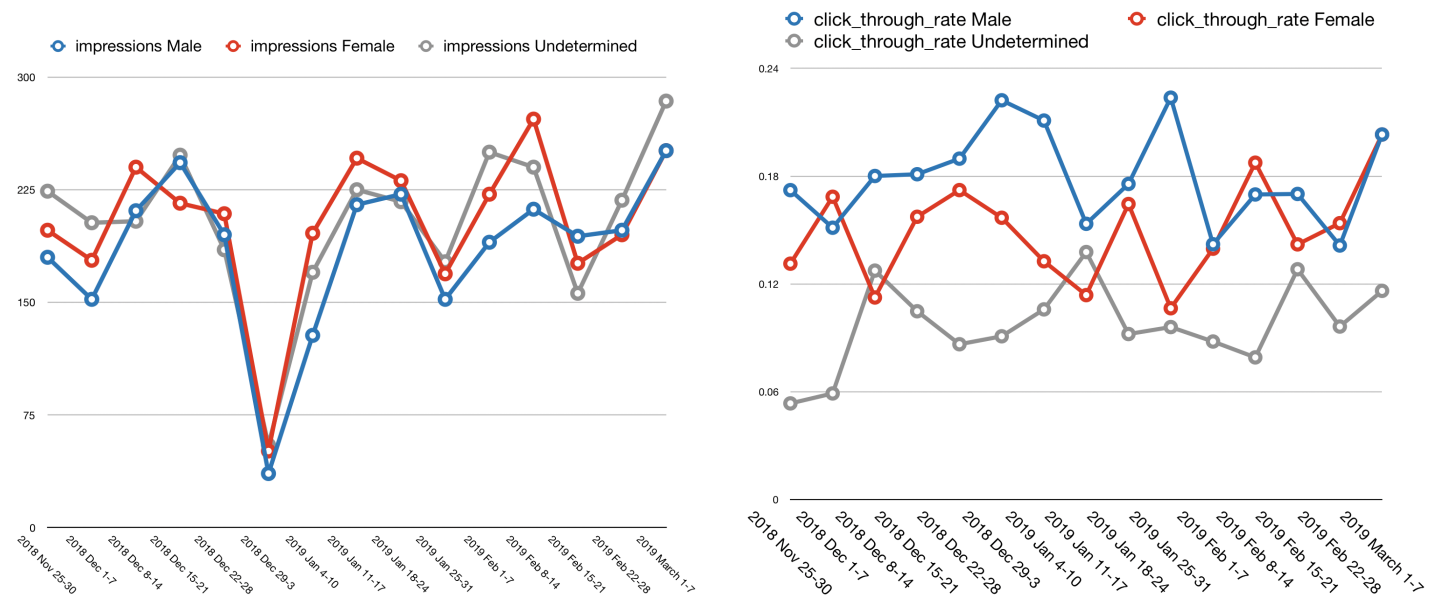

Figure 6: Gender-based segmentation.

A second example concerns the segmentation by age: Figure 7 shows that for respondents between 18 and 54 years old the number of clicks and impressions generally increase with age, although the 55-64 and 65+ age groups have lower counts in both categories.

Finally, it is worth noting here that besides Google Adwords campaigns (data from which were accessible during ESGI144), we also could use Facebook Ads and Google Contextual Ads where we can take into account different interests of users and also use pictures and videos in advertisements. 

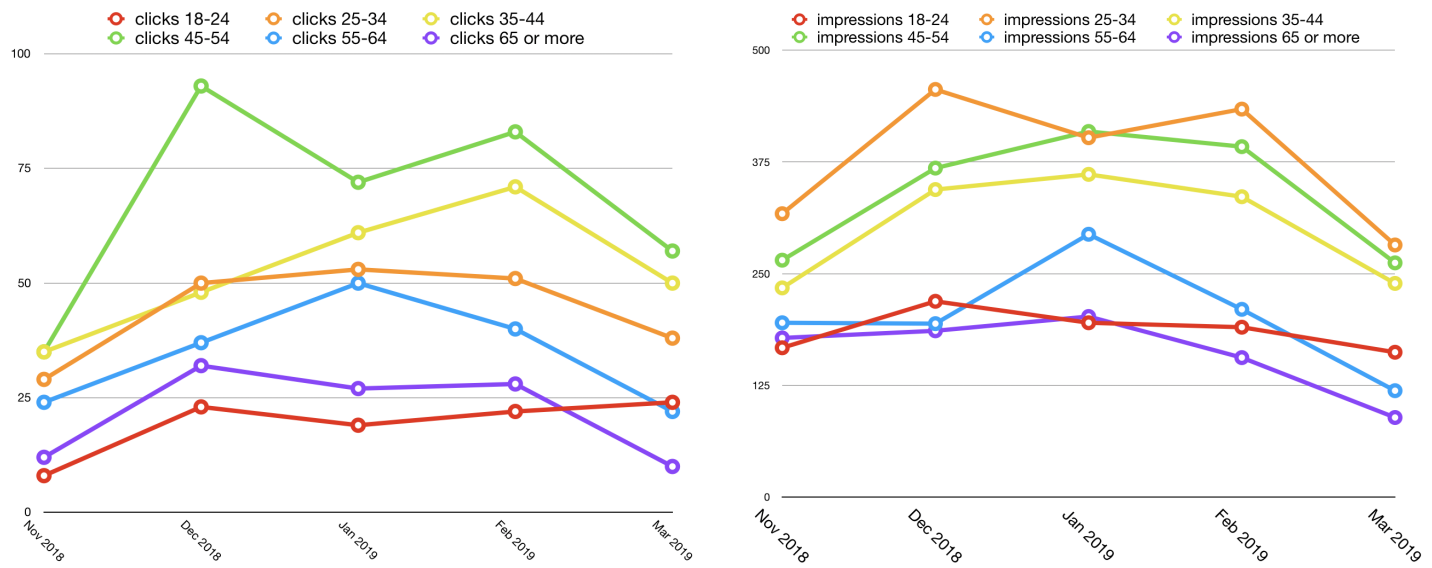

Figure 7: Age-based segmentation.

\section{Theoretical model for profit}

Consider the scenario of designing an offer (new ad campaign) for a particular segment of the population. In particular, what is involved in determining the best discount to offer? A discount of $0 \%$ will not encourage users to respond to an ad, but a discount of $100 \%$ will not make the hotel any money even if people do book reservations. There should be at least one local maximum of the profit as a function of the discount.

A basic model of the hotel's profit in this scheme is

$$
\begin{aligned}
\text { Profit } & =\left(\begin{array}{c}
\text { Conv. } \\
\text { rate }
\end{array}\right) \times\left(\begin{array}{c}
\# \text { of } \\
\text { clicks }
\end{array}\right) \times\left(\begin{array}{c}
\text { Revenue } \\
\text { per conv. }
\end{array}\right) \\
& -\left(\begin{array}{c}
\text { Conv. } \\
\text { rate }
\end{array}\right) \times\left(\begin{array}{c}
\# \text { of } \\
\text { clicks }
\end{array}\right) \times\left(\begin{array}{c}
\text { Discount } \\
\text { per conv. }
\end{array}\right) \\
& =\left(\begin{array}{c}
\# \text { of } \\
\text { conversions }
\end{array}\right) \times\left(\begin{array}{c}
\text { Revenue } \\
\text { per conv. }
\end{array}\right)-\left(\begin{array}{c}
\# \text { of } \\
\text { conversions }
\end{array}\right) \times\left(\begin{array}{c}
\text { Discount } \\
\text { per conv. }
\end{array}\right) \\
& =\left[\left(\begin{array}{c}
\text { Revenue } \\
\text { per conv. }
\end{array}\right)-\left(\begin{array}{c}
\text { Discount } \\
\text { per conv. }
\end{array}\right)\right] \times\left(\begin{array}{c}
\# \text { of } \\
\text { conversions }
\end{array}\right)
\end{aligned}
$$

Denoting the variables by

- $P$ - profit

- $R$-revenue per conversion (for a particular offer to a particular segment)

- $D$ - discount per conversion (for a particular offer to a particular segment)

- $C$-number of conversions as a function of discount, 
this becomes

$$
P(D)=(R-D) \cdot C(D) .
$$

Note that $P$ and $C$ also depend on the population segment, as does $R$ (e.9., families spend more on each reservation than single individuals do). However, the goal here to is chose the best discount for an ad campaign already targeted to a specific segment of the population, so this dependence on segment is not an issue.

\subsection{Optimisation of profit}

Recall the profit model from Equation 1:

$$
P=(R-D) \cdot C,
$$

where $P$ and $C$ are functions of $D$. When $\mathrm{d} P / \mathrm{d} D=0$, we have

$$
\begin{aligned}
0 & =R \frac{\mathrm{d} C}{\mathrm{~d} D}-C-D \frac{\mathrm{d} C}{\mathrm{~d} D} \\
C & =(R-D) \frac{\mathrm{d} C}{\mathrm{~d} D} \\
\frac{C^{\prime}(D)}{C(D)} & =\frac{1}{R-D}
\end{aligned}
$$

In practice, checking whether a given $D$ satisfies Equation 2 can be easier than checking whether $P^{\prime}(D)=0$ directly.

Using Equation 1, the second derivative of profit can be calculated as

$$
\begin{aligned}
P(D) & =(R-D) \cdot C(D) \\
P^{\prime}(D) & =\left[(R-D) \cdot C^{\prime}(D)\right]-C(D) \\
P^{\prime \prime}(D) & =\left[(R-D) \cdot C^{\prime \prime}(D)-C^{\prime}(D)\right]-C^{\prime}(D)
\end{aligned}
$$

that is,

$$
P^{\prime \prime}=(R-D) C^{\prime \prime}-2 C^{\prime}
$$

\subsection{Hypothetical conversion models}

Equation 2 holds for any nonzero function $C(D)$ giving the number of conversions as a function of the discount. Here we explore how different classes of functions $C(D)$ give rise to different critical points of the profit function $P(D)$. 


\subsubsection{Linear conversion function}

Suppose that the number of conversations is linear with respect to the discount. Denoting $C_{0}=C(0)$, we are assuming

$$
C(D)=C_{0}+a D \text {. }
$$

For what values of $D$ does the profit $P(D)$ have a critical point? Using Equation 2, this occurs when

$$
\begin{aligned}
\frac{C^{\prime}(D)}{C(D)} & =\frac{1}{R-D} \\
\frac{a}{C_{0}+a D} & =\frac{1}{R-D} \\
a R-a D & =C_{0}+a D \\
\left(C_{0}-a R\right)+2 a D & =0
\end{aligned}
$$

Therefore $P$ has only one critical point, and it occurs when $D=D^{*}=\frac{C_{0}-a R}{2 a}$.

The Second Derivative Test should tell us whether this critical point is a maximum or minimum of $P$. Plugging $C^{\prime \prime}(D)=0$ and $C^{\prime}(D)=a$ into Equation 3, we have

$$
P^{\prime \prime}\left(D^{*}\right)=\left(R-\frac{C_{0}-a R}{2 a}\right)(0)-2(a)=-2 a,
$$

so the critical point is a maximum of $P$ (that is, $P^{\prime \prime}\left(D^{*}\right)<0$ ) if and only if the parameter $a$ in the model $C(D)=C_{0}+a D$ is positive, which would mean that the number of clicks strictly increases as the discount increases. This is as expected.

\subsubsection{Quadratic conversion function}

Suppose that the number of conversations is quadratic with respect to the discount. Denoting $C_{0}=C(0)$, we are assuming

$$
C(D)=C_{0}+a D+b D^{2} .
$$

For what values of $D$ does the profit have a critical point? Using Equation 2, this occurs when

$$
\begin{aligned}
\frac{C^{\prime}(D)}{C(D)} & =\frac{1}{R-D} \\
\frac{a+2 b D}{C_{0}+a D+b D^{2}} & =\frac{1}{R-D} \\
(R-D)(a+2 b D) & =C_{0}+a D+b D^{2} \\
R a-a D+2 b R D-2 b D^{2} & =C_{0}+a D+b D^{2} \\
3 b D^{2}+2 a D-2 b R D+C_{0}-R a & =0
\end{aligned}
$$


Therefore $P$ has two critical points, occurring when

$$
D^{*}=\frac{b R-a \pm \sqrt{a^{2}-3 b C+a b R+b^{2} R^{2}}}{3 b} .
$$

The Second Derivative Test should tell us whether each critical point is a maximum or minimum of $P$. Plugging $C^{\prime \prime}(D)=2 b$ and $C^{\prime}(D)=a+2 b D$ into Equation 3, we have

$$
P^{\prime \prime}\left(D^{*}\right)=\left(R-D^{*}\right)(2 b)-2\left(a+2 b D^{*}\right)=2(b R-a)-6 b D^{*}
$$

and therefore $P^{\prime \prime}<0$ (a maximum of $P$ ), requires

$$
\begin{array}{rlrl}
0 & >2(b R-a)-6 b D^{*} \\
6 b D^{*} & >2(b R-a) \\
D^{*} & >\frac{b R-a}{3 b} \text { if } b>0, &
\end{array}
$$

\subsubsection{Exponential conversion function}

Suppose that the number of conversions is exponential with respect to the discount. Denoting $C_{0}=C(0)$, we are assuming

$$
C(D)=C_{0} e^{a D}
$$

For what values of $D$ does the profit have a critical point? Using Equation 2, this occurs when

$$
\begin{aligned}
\frac{C^{\prime}(D)}{C(D)} & =\frac{1}{R-D} \\
\frac{a C_{0} e^{a D}}{C_{0} e^{a D}} & =\frac{1}{R-D} \\
a & =\frac{1}{R-D}
\end{aligned}
$$

Therefore $P$ has only one critical point, and it occurs when $D=D^{*}=R-\frac{1}{a}$.

The Second Derivative Test should tell us whether this critical point is a maximum or minimum of $P$. Plugging $C^{\prime}(D)=a C_{0} e^{a D}$ and $C^{\prime \prime}(D)=a^{2} C_{0} e^{a D}$ into Equation 3, we have

$$
\begin{aligned}
P^{\prime \prime}\left(D^{*}\right) & =\left(R-\left(R-\frac{1}{a}\right)\right) a^{2} C_{0} e^{a D}-2 a C_{0} e^{a D} \\
& =\left(\frac{1}{a}\right) a^{2} C_{0} e^{a D}-2 a C_{0} e^{a D} \\
& =a C_{0} e^{a D}-2 a C_{0} e^{a D} \\
& =-a C_{0} e^{a D}
\end{aligned}
$$

Since $C_{0}>0$, this critical point is a maximum of $P$ if and only if the parameter $a$ in the model $C(D)=C_{0} e^{a D}$ is positive, which would mean that the number of clicks strictly increases as the discount increases. 


\subsection{Dynamic pricing}

The present problem also concerns dynamic pricing. Dynamic pricing is a strategy of varying the price for a product to reflect changing market conditions, aiming to yield greater demand. In dynamic pricing [22], the function to analyse is the demand $d$ as a function of the price $p$. It is customary to assume that $d(p)$ is a decreasing function, which merely means that if a product is sold for a higher price, the demand would decrease. Mathematically, this has a simple consequence: the price can be solved as a function of demand. Therefore, this seemingly alternative approach to the previous sections where we viewed the price $p$ as a function is well within our present context. Although determining the actual demand function $d(p)$ can be a challenging task, there had been some efforts to compute and analyse such functions [2].

In this subsection, we analyse the logit function [2].

$$
d(p)=\frac{C e^{-(a+b p)}}{1+e^{-(a+b p)}}
$$

$C, a$ and $b$ are constants to be determined. The price $p$ above can be computed as a function of demand.

$$
a+b \cdot p(d)=\log \left(\frac{C-d}{d}\right) .
$$

In this model, the price already encompasses the discount, that is, $p(d)$ is the final price.

Note that since the price is a function of demand, conceptually, it does not make sense to determine the demand that maximises the price, but optimising $d(p)$ makes sense.

By computing the derivative of $d(p)$,

$$
\begin{aligned}
d^{\prime}(p) & =-(a+b p) \frac{C e^{-(a+b p)}}{1+e^{-(a+b p)}}+(a+b p) \frac{C e^{-(a+b p)}}{\left(1+e^{-(a+b p)}\right)^{2}} \\
& =-(a+b p) \frac{C e^{-(a+b p)}}{1+e^{-(a+b p)}}\left(1-\frac{1}{1+e^{-(a+b p)}}\right),
\end{aligned}
$$

we see that $p=-a / b$ is the unique critical point.

Although this simple model does not depict the full complexity of dynamic pricing-the function $d$ should also depend on time and must reflect relevant changes in market conditions-it is a frequently-used model [2] and is still important. The most challenging part is that the constants $a, b$, and $C$ must be measured in each cluster also as functions of time in practice. 
Targeting Ads and Inferring Demographics

ESGI144

\section{Decision-making processes}

\subsection{Bayesian updating}

A Bayesian updating scheme takes the usual expression for Bayesian inference of updating the probability for a hypothesis based on a prior probability, as more evidence becomes available. In the case of probability distributions and dynamic updating, Bayesian updating is represented by a recurrence relation in which the posterior probability is updated by the new information.

In the case of modelling the probability density function for profit based on a discount (see Equation 1 and Section 3), the scheme would have a prior estimated from previous compaigns and would be updated by information from the campaign.

\subsection{Feedback loop}

The process of creating new offers to specific segments can be done repeatedly, updating the segments and offers as new data are gathered on existing ad campaigns.

An outline of the process is as follows:

Step 1. Use machine learning to create clusters based on the PMS data (see Section 2.1).

Step 2. If the hotel does not collect consistent demographic data, run an ad campaign using the Customer IDs so that Google's data can help determine the demographics of a cluster, that is, $\mathbb{P}(S \mid$ cluster $)$ for each segment $S$.

Step 3. Create individual profiles to estimate the probability that a customer fits a particular demographic (this will allow us to create targeted ads). We estimate the vector of probabilities of belonging to one of the demographic segments, $\mathbb{P}(S)$, for each user in the PMS data via the formula

$$
\begin{aligned}
\mathbb{P}(S) & =\sum_{C_{k}} \mathbb{P}\left(S \text { and } C_{k}\right) \\
& =\sum_{C_{k}} \mathbb{P}\left(S \mid C_{k}\right) \cdot \mathbb{P}\left(C_{k}\right) .
\end{aligned}
$$

Here we assumed that all people of a particular demographic react to ads in approximately the same way; therefore, we can get a demographic profile for each cluster. For example, if for a particular cluster we get that $70 \%$ of women and $30 \%$ of men clicked on the ad, then we assume that $70 \%$ of this cluster are women and 
Targeting Ads and Inferring Demographics

$30 \%$ are men. That way we get all the probabilities $\mathbb{P}\left(S \mid C_{k}\right)$, which in this case would be

$$
\mathbb{P}\left(\text { the user is a woman | the user is in cluster } C_{k}\right)=0.7 \text {. }
$$

Step 4. Create new ad campaigns including specialised offers based on new information from demographics, and create offers based on the discount rate (see Section 3.1).

Step 5. Collect data on the success rate of the targeted offers.

Step 6. Send "successful" offers by e-mail.

Note: In Step 1 it is possible to create clusters by hand using the most important features for an offer making process (for example, the number of children or adults, booking in advance, season, etc.). The only condition is that groups have to be large enough. Then we can "reverse" Step 3 and make a prediction of new users of particular demographic segment being in a certain cluster. This way we can target and re-target new users who have not yet stayed at the hotel. This will be especially useful for Facebook and Google Contextual Ads.

\section{Further ideas}

Several other models and algorithms were suggested but not fully developed during the course of ESGI144. Here we present these ideas with the hope that others will find them useful and continue our work.

\subsection{Click-based profit models}

Google Adwords charges based on the number of clicks on an advertisement [9], while the hotel generates revenue only from conversions (bookings). For larger ad campaigns, this distinction may be significant, and to model this we decompose the number of conversions in Equation 1 as number of clicks multiplied by the conversion rate. We also subtract a term that incorporates the Google ad fee. Thus our revised profit model is

$$
P=C \ell \cdot C v r \cdot(R-D)-C \ell \cdot A,
$$

where the variables are

- $P$ - profit

- $C \ell$-number of clicks 
- Cvr - conversion rate

- $R$-revenue per conversion

- $D$ - discount per conversion

- $A$ - ad fee per click.

Since every variable except $A$ depends on the discount $D$, the Chain Rule gives the derivative $d P / d D$ as

$$
P^{\prime}=C \ell \cdot C v r \cdot\left(R^{\prime}-1\right)+C \ell \cdot C v r^{\prime} \cdot(R-D)+C \ell^{\prime} \cdot C v r \cdot(R-D)-C \ell^{\prime} \cdot A .
$$

Trying to solve $P^{\prime}(D)=0$ analytically is unreasonable, but numerical approximations can still be used (see [27] for examples).

\subsection{Ad campaign strategies}

In this section we propose a few more strategies for creating an ad campaign and getting good segmentation of clients with respect to demographics.

The first strategy is as follows.

1. Fix the set of offers from the hotel, and for each offer create three (heuristic) slightly different ads.

2. Run all ads for all users.

3. Analyse the "best performing" demographics and "best performing" offers and ads.

4. Target chosen groups with chosen ads with higher price for an ad.

This procedure can be viewed as a loop. First we start with more general offers suited for all demographics and see which demographics perform best. Then we adjust ads, adding more specifics and testing ads using other proposed strategies (for example, adding other features to an offer or testing different discounts).

The second strategy, a greedy one, concerns the demographic segments we found in Step 4 of the process in Section 4.2. Here we want to test how slight changes in one particular campaign will improve the performance of an ad. We don't want to test all the possibilities for changes that could be made to an ad.

1. Start with a simple baseline offer. Run an ad campaign.

2. Run three campaigns (again heuristics) with one additional element added to the baseline offer. 
3. Choose the best offer and run two more campaigns with one more addition each.

4. Choose the best offer. If there are no improvements, choose the best offer from previous steps.

See Figure 8 for an example of how we can construct a greedy procedure for searching in a state of ads.

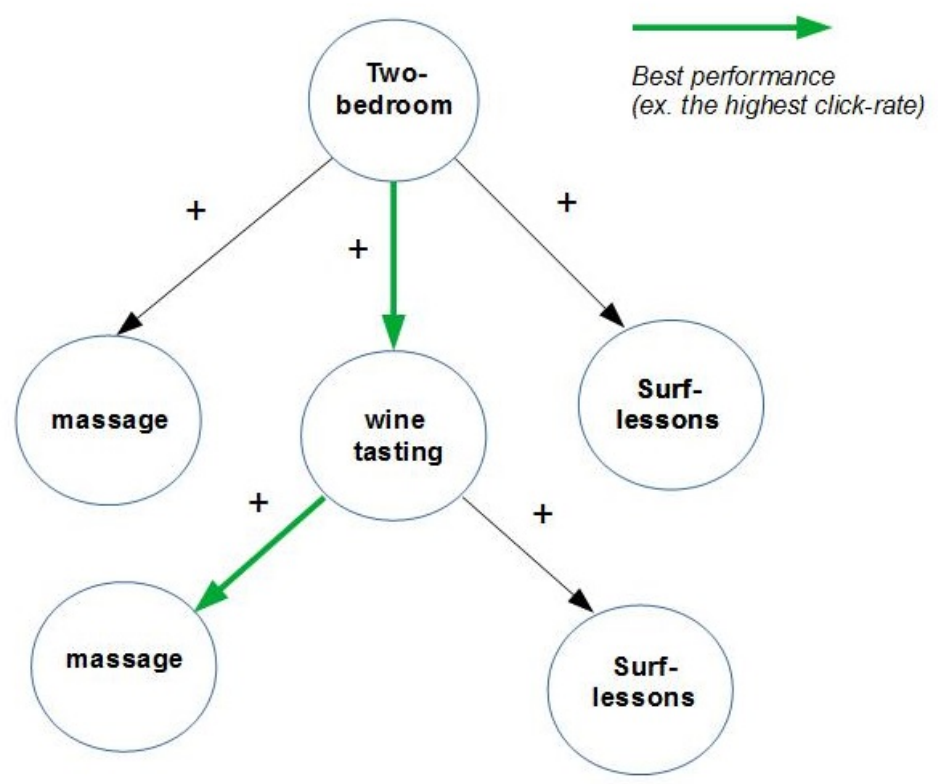

Figure 8: An example of a greedy procedure for a baseline offer (two-bedroom) and three additional offers. The best offer in this case is two-bedroom + wine-tasting + massage.

Greedy algorithms are the ones that construct an object one step at a time, at each step choosing the locally best option. Greedy procedures have a few advantages: they are usually easy to describe and implement, and they are time-efficient. On the other hand, they are sometimes hard to design, meaning that it is hard to find the right approach of building such an algorithm. But when it is found, then it is simple to design. Additionally, it is sometimes hard to verify the correctness of the algorithm, that is, to prove that choosing locally best options will give a globally best solution. For more information on greedy algorithms, see, for example, [7]. 


\subsection{Automation algorithm}

Ideally, we want an algorithm that will traverse the space of all advertisements intelligently to select the best ads to offer. The idea is to establish a space of states (of ads) where you whether arrange ads (offers) in a grid of cells or define some kind of distance between two offers which should have interpretation in real life. In the case of the grid adjacent cells have only one difference (one change) and the intelligent agent can only move to a neighbor cell. See Table 1. So here we control how click rate changes with one small change of an offer. While the campaign is improving we keep updating an ad looking for a better version but at some point the output of a new campaign is going to be worse than at the previous step. And at this point the question is when to stop exploring and pick the best ad.

\begin{tabular}{|c|c|c|c|c|}
\hline $2 b+a_{7}+a_{5}$ & $2 b+a_{1}+a_{5}$ & $2 b+a_{5}$ & $2 b+a_{5}+a_{2}$ & $2 b+a_{5}+a_{8}$ \\
\hline $2 b+a_{7}+a_{1}$ & $2 b+a_{1}+a_{4}$ & $2 b+a_{1}$ & $2 b+a_{1}+a_{2}$ & $2 b+a_{1}+a_{8}$ \\
\hline \hline $2 b+a_{7}$ & $2 b+a_{4}$ & $2 b$ & $2 b+a_{2}$ & $2 b+a_{8}$ \\
\hline \hline $2 b+a_{7}+a_{4}$ & $2 b+a_{3}+a_{4}$ & $2 b+a_{3}$ & $2 b+a_{3}+a_{2}$ & $2 b+a_{2}+a_{8}$ \\
\hline $2 b+a_{7}+a_{6}$ & $2 b+a_{6}+a_{4}$ & $2 b+a_{6}$ & $2 b+a_{3}+a_{6}$ & $2 b+a_{2}+a_{6}$ \\
\hline
\end{tabular}

$2 b$ - two-bedroom, baseline offer;

$a_{i}$ - additional offers by a hotel (for example, free massage, wine tasting, free access to a gym, excursion, sauna) for any $i=1,2, \ldots, 8$

Table 1: An example of a grid of offers with one baseline offer and eight additional offers.

Some of possibilities on how to explore this space are:

- Penalise the cells with worse performance so the agent is unwilling to go back there;

- Start with equal probabilities to move up, down, right or left. Then start (somehow) updating the probabilities so that the probability to go to a worse performing ad becomes much less (but not zero so we still give a chance to not successful ads);

- Mark cells as + and - after we start moving through the space of ads. If we go to a better performing cell mark it + and a current one as -, in the case where we go to a worse performing cell, mark it - and a current one as +. Here we might allow the agent jump through a marked cell. Then not all pluses and minuses will be equivalent. As a stop point we can establish a certain threshold for a ratio of pluses and minuses.

As mentioned previously, the space of ads can be organised in a different way. On this space we can define some kind of distance where the ads which have fewer differences are 
closer to each other than ads with more differences. As such distance Hamming distance can be used. Each ad will be represented by a binary vector of length $n$, where $n$ is the number of possible hotel offers which can be included in one offer (ad). Therefore, in the case from Table 1 with one baseline and 8 additional offers ads will be represented by 9 (if we want to test a change for a baseline offer, e.g. from two-bedroom to three-bedroom) or 8-dimensional binary vectors with a coordinate $i$ equal to 1 if ad contains an offer $a_{i}$ and 0 if not. Hamming distance $D(x, y)$ between two points $x$ and $y$ is defined in the space of $q^{n}$ (in our case $q=2$ ) points as the number of coordinates for which $x$ and $y$ are different [10].

\subsection{DFO}

Ideally we want to maximise a function that takes as its inputs user characteristics and ad features and outputs a conversion rate score.

Given that we do not have access to the derivative of this function, and it is expensive to evaluate (in fact, it requires an add campaign to evaluate!), it might be of interest to consider derivative-free optimisation strategies (DFOs), in order to determine what "point" (ad features and user profiles) to choose next. Examples of DFOs include simulated annealing [15] and Bayesian optimisation [20] (the latter requires some prior understanding of how features interact and affect the objective function). These approaches require some way to determine the ad and user features.

\subsection{Hidden Markov Models}

A Hidden Markov Model (HMM) models a process that is assumed to depend on a Markov process of transitions between un-observable (hidden) states. Given a series of observations of observable states, it is possible to construct the best emission (hidden-hidden) probabilities and transmission (hidden-observable) to fit these observations. Consider for example if one wished guess whether or not a friend is hungry (hidden) based on their mood (observable); happy or angry. Over a series of time periods the mood of the friend is observed, and these observations are used to determine the probability of transitioning between the states of hungry and not hungry, and the likelihood both states have in resulting in each mood. The left-hand side of 9 illustrates this concept, where the solid arrows indicate observed transitions and the dashed and dotted lines indicate the transitions whose probabilities are to be estimated. 

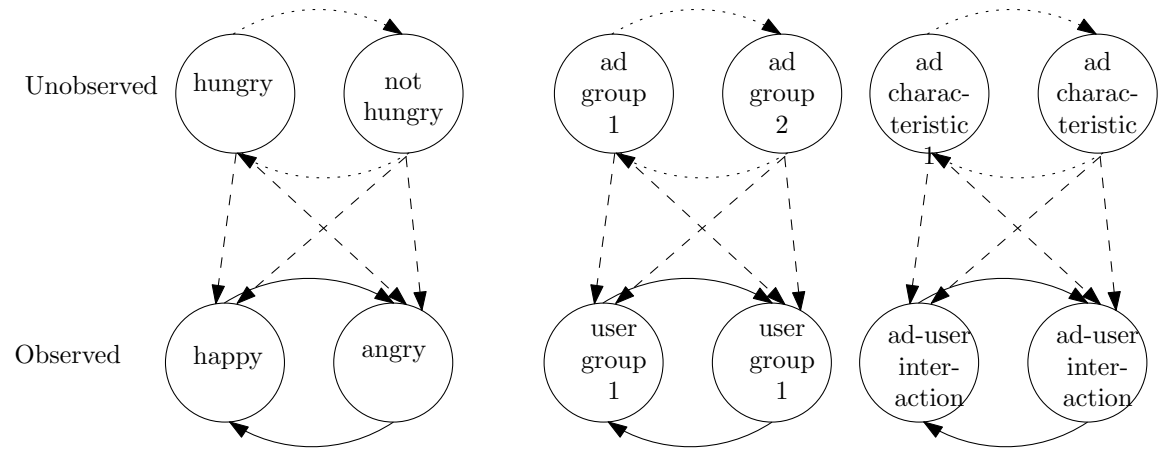

Figure 9: Replacing the notion of transition within the context of a Hidden Markov Model with that of similarity could help group adverts that have similar impact on user groups

The parallel to the hotel problem is that there exist certain characteristics of ads (discounted prices, room upgrades, complimentary extras) and users (such as age, family structure, sense of adventure), however in many cases we can only observe the interactions between them; i.e. when a particular user clicks a particular ad. This line of thinking may be of interest for deriving information about the background characteristics of users and ads.

A fundamental difference between the two problems is that in the context of ad generation there is not the same notion of transition; in general we have assumed that the characteristics of ad and users do not change over the time period of interest. However, it might be possible to replace notions of transition with similarity and make some progress that way. For example, determining similarity between ad groups based on the similarity of users that respond to them (centre of 9), or gaining insight into the latent features of ads based on observed interactions (right-hand side of 9).

\subsection{Deep learning}

Given enough data, we might hope to train a neural network to predict whether a user will interact with a given ad, based on the features of both the ad and the user. However, as we gather data, our knowledge of the features of individuals may change. The following deep learning approaches have been used with recurrent neural networks (RNNs) when features are missing or change slowly over time.

The authors of [18] use a combination of imputation, adding a missing variable indicator feature, and feature design to deal with the problem of missing data with RNNs. The underreview paper [26] proposes a method for features that change much more slowly than other features. This could be used for predicting change in seasonal demand, for example, when customers start booking summer holidays. 
However, both of these methods are targeted towards time series problems. For the hotel problem, the temporal component comes from users reacting over time, and so the idea of finding patterns depending on what has come before may not be appropriate; we do not have a reason to assume that there are patterns in the conversion rates over multiple days.

\subsection{Recommendation systems approach}

A Recommendation System usually refers to a system which is capable of predicting users' preferences for a number of products or items (it gives a list of items that a user is likely to buy, rate positively, or otherwise interact with). Data in this kind of problem are represented by a user-item matrix, which is usually sparse, where a cell contains information about how and if one particular user interacted with one particular item (e.g., did or did not buy the item, rated it according to a certain scale). Sometimes the matrix may also contain additional features about items. Therefore, the first thing to do is to define how our matrix will look.

Here we propose a few options of user-item matrices that could be used:

- A user-offer matrix for internal advertisement. If we have a large hotel or a chain of hotels with a history of shared regular clients and many offers to choose from, we can build a client-offer matrix. One cell will contain information about a single client and their interaction with a particular offer. Hence, the recommendation system will only work for people who are already clients of the hotel. To those people, we can send targeted e-mails with a few top offers from a recommendation system.

- A user-offer matrix for external advertisement. Again this probably would work better for chains of hotels with many offers. Here, instead of one user we will target small group of users and see how they react to an ad or an offer. The matrix is (smallgroup-of-users)-(ad-for-an-offer). One cell will contain information about ad click-rate or conversions for a small group of users who have been shown the ad. Here we can find groups of users with similar preferences and pay more for offers they are more likely to consider. This matrix could also potentially be combined with the matrix from the previous example to compare new user groups with old clients.

- A user-ad matrix. As in the previous example it would be better to have users combined into small groups. The matrix will be (small-group-of-users)-ad, where a single cell will contain information about ad click-rate. It can be used for smaller hotels, when we want to test ads, not offers, so for one offer we can create dozens of ads with different words, order of words, pictures, etc. This is a way to test Facebook Ads or Google Contextual Ads because with these services you can show ads to people who 
didn't enter a web search query relating to an offer and you can divide people by interests rather than by features like gender or age.

In all cases, we could have binary matrices and only look at whether a client or a group of users interacted with an offer or an ad. In the first case, we are only interested in whether a person bought an offer or didn't; in the second and the third cases we are interested in whether users reacted to an ad (clicked on it or booked a room). Instead of binary matrices, we could have matrices with numbers in a range (e.9., from 1 to 5 or 1 to 10). In the first case such information would clients' ratings of offers, and in the latter two cases we could create our own scale measuring how "good" various click-rates are for business. This should be supported by empirical evidence and could be adjusted over time.

There are basically two main approaches to building a recommendation systems: Contentbased and Collaborative filtering. There is also some literature regarding Hybrid algorithms that combine both approaches (see $[8,13,3]$ ).

The content-based approach uses some additional features of the items, so in addition to a user-item matrix mentioned above we also need to have some good amount of information about items. In our case, offers or ads can have features such as country or area, season, time of stay, types of activities included, etc. The recommendation process is based on the content similarity of items. For example, suppose we had only one feature, "type of activity". If the user previously had trips of one particular type of activity which they liked, and if we have another offer with this same type of activity, then our recommender will show this offer to the user. To understand these preferences, the user's profile is built. It is represented by a matrix of weights corresponding to importance or rating of different features for the user.
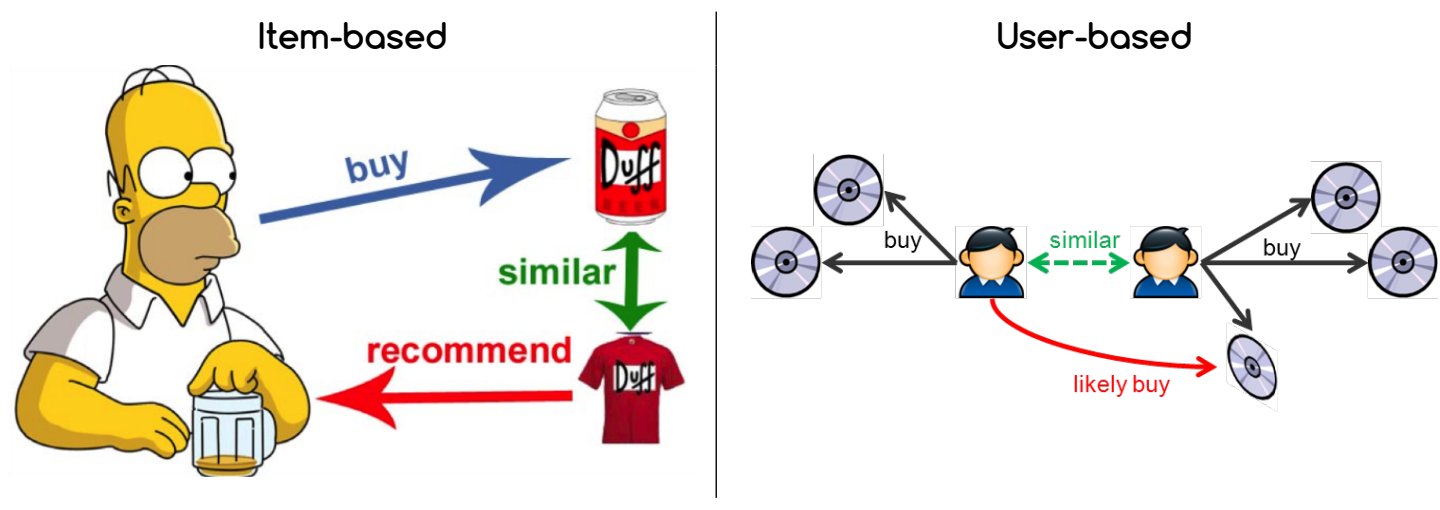

Figure 10: Illustrations of item-based and user-based collaborative filtering.

Collaborative filtering (CF), on the other hand, does not need any additional information about users or items except historical data. Collaborative filtering can be User-based or Item-based; see Figure 10 for an illustration of the difference (images are from [11] and [12]). 
In the User-based approach, we calculate similarity between a user we want to make a prediction for and all other users, then we choose a few closest users in terms of preferences and take the weighted average of their ratings. Here it is also possible to avoid the bias that some users generally tend to rate everything higher than other users or click on ads more often than other users. To calculate similarities between two users, generally two measures of similarity are used: Pearson Correlation, Cosine Similarity, and Adjusted Cosine Similarity [24].

In the Item-based approach, we calculate similarities between items based on how they were rated by users. The recommendation is then made by calculating weighted averages of ratings on the items most similar to the items rated or liked by the target user.

Both approaches have their advantages and disadvantages. The Content-based approach needs a sufficient amount of information about items, but when we have it we can avoid the "new-item problem" that we will encounter with Collaborative filtering. Content-based approaches also carry a risk of getting stuck around the same type of products the user has already consumed. However, the Content-based approach is transparent and logically explainable, whereas CF is not.

Collaborate filtering does not handle sparsity of data well and is not easily scaleable, but Matrix Factorisation can be used to mitigate these problems. We decompose our huge and sparse matrix into low-dimensional matrices of new latent features which represent users' preferences and items' characteristics. The intuition behind these new matrices is that there are some higher-level types of preferences than we can see from particular choices made by the user. For example, if the user previously chose Kenya and Tanzania as trip destinations, then the higher-level preference might be African countries or Saforis. This factorisation problem can be solved by Alternating Least Squares, which is an iterative optimisation algorithm to approximate Singular Value Decomposition. It is also possible to use deep learning techniques for matrix factorisation.

There exists a huge amount of literature on the subject of Recommender Systems. Here we provide some references on Content-based approaches [21], Collaborative filtering [25], and in particular item-based filtering [24, 17], which tends to be a more popular choice of CF. We also provide a reference for Recommender Systems for binary matrices [19] and some general guidelines [28] on developing Recommender Systems, including which questions to answer before building them. 


\section{References}

[1] David Barber. Bayesian Reasoning and Machine Learning. Cambridge University Press, 2012.

[2] Bayoumi, Saleh, Atiya, Aziz. "Dynamic pricing for hotel revenue management using price multipliers". J. Revenue Pricing Manag. (2013) 12: 271.

[3] A. Bellogin, I. Cantador, F. Rubio, P. Castells, E. Chavarriaga. "An Empirical Comparison of Social, Collaborative filtering, and Hybrid Recommenders". ACM Transactions on Intelligent Systems and Technology, 4(1), February 2013, 1-29.

[4] Christopher M. Bishop. Pattern Recognition and Machine Learning. Springer, New York, 2006.

[5] L. Cantoni, M. Faré, A. Inversini, V. Passini. "Hotel Websites and Booking Engines: A Challenging Relationship" in Law R., Fuchs M., Ricci F. (eds) Information and Communication Technologies in Tourism. Springer, Vienna, 2011.

[6] Jason Clampet. "Everything You Wanted to Know About the Hotel Industry's Gripes Against OTAs". Skift, 25 April 2016.

[7] Thomas H. Cormen, Charles E. Leiserson, Ronald L. Rivest, Clifford Stein. "Ch. 16: Greedy algorithms" in Introduction to Algorithms, MIT Press and McGraw-Hill, 2001.

[8] G. Geetha, M. Safa, C. Fancy, D. Saranya. "A Hybrid Approach using Collaborative filtering and Content based Filtering for Recommender System". Journal of Physics: Conference Series 1000(1):012101, April 2018.

[9] Google. Get Results with an Advertising Budget That Works for You. Retrieved 22 March 2019 from https://ads.google.com/intl/en_us/home/pricing/

[10] R. .W. Hamming, "Error detecting and error correcting codes". The Bell System Technical Journal. 29 (2): 147-160.

[11] Handaru. "Build Recommendation Engine Using Graph". ticket-dev, Medium. 11 June 2016. https://link.medium.com/zGoOFEUmgW

[12] Ricky Ho. "Recommendation Engine Models". DZone. 2 November 2011. https://dzone. $\mathrm{com} /$ articles/recommendation-engine-models

[13] Z. Huang, W. Chung, H. Chen. "A graph model for e-commerce recommender systems". Journal of the American Society for Information Science and Technology, 55(3), February $2004,259-274$. 
Targeting Ads and Inferring Demographics

ESGI144

[14] Martin Kelly. "OTAs Increase Market Share at Supplier's Expense". Travel Trends, 24 February 2017.

[15] Scott Kirkpatrick, Daniel Gelatt, Mario Vecchi. "Optimization by simulated annealing". American Association for the Advancement of Science, 220 (1983), 671-680.

[16] Jacques Levy-Bonvin. "Hotels | A Brief History". Hospitality Net, 15 December 2003.

[17] G. Linden, B. Smith, J. York. "Amazon.com recommendations: item-to-item collaborative filtering". IEEE Internet Computing. 7(1), January 2003, 76-80.

[18] Zachary Lipton, David Kale, Randall Wetzel. "Modeling missing data in clinical time series with RNNs". Machine Learning for Healthcare, 2016.

[19] C. Miranda, A. M. Jorge. "Incremental collaborative filtering for binary ratings". Web Intelligence Conference Proceedings, IEEE Computer Society. (2008), 389-392.

[20] Jonas Mockus. "Bayesion approach to global optimisation: theory and applications". Springer Science \& Business Media. 37 (2012).

[21] Michael J. Pazzani, Daniel Billsus. "Content-based recommendation systems", The Adap. Web (2007), 325-341.

[22] Robert Lewis Phillips. "Pricing and Revenue Optimization". Stanford University Press. 355.

[23] RoomSage. "Advertising platform, personalized marketing | RoomSage". Retrieved 22 Morch 2019 from https://roomsage . com.

[24] B. Sarwar, G. Karypis, J. Konstan, J. Riedl. "Item-based collaborative filtering recommendation algorithms". Proceedings of the 10-th International Conference on the World Wide Web (2001), 285-295.

[25] J. Ben Schafer, D. Frankowski, J. Herlocker, S. Sen. "Collaborative filtering Recommender Systems". The Adaptive Web, Springer: Berlin/Heidelberg, Germany, January 2007, 291324.

[26] Alexander Stec, Diego Klabjan, Jean Utke. "Unified recurrent neural network for many feature types". 2018. Pre-print at arXiv:1809.08717.

[27] J. Stoer, R. Bulirsch. "Chapter 5: Finding Zeros and Minimum Points by Iterative Methods" in Introduction to Numerical Analysis. Springer, New York. 1993.

[28] L. Terveen, W. Hill. "Beyond recommender systems: helping people help each other". $\mathrm{HCl}$ in the New Millennium, J. Carroll, Ed. Boston, MA, USA: Addison-Wesley, (2001), 487-509. 